\title{
IVIG pluripotency and the concept of Fc-sialylation: challenges to the scientist
}

\section{Stephan von Gunten, Yehuda Shoenfeld, Miri Blank, Donald R. Branch, Tchavdar Vassilev, Fabian Käsermann, Jagadeesh Bayry, Srini Kaveri and Hans-Uwe Simon}

Intravenous immunoglobulin (IVIG) predominantly consists of pooled polyclonal IgG from thousands of donors and it is used therapeutically for several autoimmune and inflammatory conditions. In a recent Review article (Intravenous immunoglobulin therapy: how does IgG modulate the immune system? Nature Rev. Immunol. 13, 176-189 $(2013))^{1}$, Schwab and Nimmerjahn discuss current insights into the immunomodulatory mechanisms of IVIG preparations. The authors promote a recently challenged concept that the sialylation of IgG Fc fragments is likely to be responsible for the therapeutic activity of IVIG, however, they fall short in their discussion of the experimental evidence that does not support this concept.

Schwab and Nimmerjahn discuss a hypothetical sialic acid-dependent pathway that involves the binding of Fc-sialylated IgG to mouse SIGNR1 and its human orthologue DC-SIGN (DC-specific ICAM3-grabbing non-integrin) that ultimately leads to the upregulation of the inhibitory Fc receptor $(\mathrm{FcR}) \mathrm{Fc} \gamma \mathrm{RIIB}$ on macrophages and dendritic cells (DCs) ${ }^{1}$. It should be noted that the expression and anatomical distribution of DC-SIGN in humans and of its orthologue SIGNR1 in mice differ considerably and, unlike humans, mice lack the activating receptor Fc $\gamma$ RIIA; hence results that are obtained in mice could be biased. Of note, in patients, upregulation of the expression of Fc $\gamma$ RIIB by IVIG could not be confirmed by gene expression profiling even in a pathological condition, Kawasaki's disease, where IVIG has a proven efficacy ${ }^{2}$. Furthermore, it has recently been shown that IVIG reciprocally regulates human pathogenic $\mathrm{T}$ helper $17\left(\mathrm{~T}_{\mathrm{H}} 17\right)$ and $\mathrm{T}_{\mathrm{H}} 1$ cells and regulatory $\mathrm{T}\left(\mathrm{T}_{\mathrm{Reg}}\right)$ cells independently of DC-SIGN and sialylated Fc fragment interactions ${ }^{3}$. In both human and mouse systems, $\mathrm{F}\left(\mathrm{ab}^{\prime}\right)_{2}$ fragments of IVIG exerted effects that were similar to intact IVIG. Investigation of the mechanism in human DCs further revealed that $\mathrm{F}\left(\mathrm{ab}^{\prime}\right)_{2}$ fragments of IVIG could directly interact with
DC-SIGN that is expressed on DCs, which induced both cyclooxygenase 2 (COX2) activation and prostaglandin E2 (PGE2) production and led to an expansion of $\mathrm{T}_{\text {Reg }}$ cell populations ${ }^{4}$.

Schwab and Nimmerjahn ${ }^{1}$ state that "IVIG enriched for sialic acid-rich glycovariants of the $\mathrm{F}\left(\mathrm{ab}^{\prime}\right)_{2}$-fragment did not show increased therapeutic activity" citing four articles. However, two of the cited articles do not include functional experiments but rather focus on aspects of human IgG sialylation and lectin fractionation. The other articles report that in a human whole blood inflammation assay, the anti-inflammatory activity of IVIG was associated with Fab sialylation and not Fc sialylation ${ }^{5}$, and that in a murine model of passive-immune thrombocytopenia no increase in platelet count was observed in response to the administration of IVIG that was enriched for sialylated $\mathrm{IgG}^{6}$. Together, the data presented in these publications do not support the proposed Fc-sialylation concept - by contrast, they rather challenge it. Indeed, another independent group has recently shown the effects of IVIG to be independent of sialylation of the Fc regions of IVIG ${ }^{7}$.

Recent evidence also challenges the concept put forth by Schwab and Nimmerjahn of a requirement for the inhibitory Fc $\gamma$ RIIB receptor in the mechanism of IVIG. Despite the claim made by Schwab and Nimmerjahn of a crucial role for Fc $\gamma$ RIIB in the Fc-sialylation concept ${ }^{1}$, independent groups recently showed - using different strains of Fc $\gamma$ RIIB-deficient mice - that IVIG-mediated beneficial effects did not require the presence of $\mathrm{Fc} \gamma \mathrm{RIIB}^{8,9}$. In contrast to $\mathrm{F} c \gamma \mathrm{RIIB}$-deficient BALB/c mice, Fc $\gamma$ RIIB-deficient C57BL/6 mice required more IVIG for the amelioration of disease. It was suggested that both strains of knockout mice should be tested and should show concordance before any definitive conclusions about the role of Fc $\gamma$ RIIB can be made. Unfortunately, although Schwab and Nimmerjahn cite some of the crucial reports regarding the role of $\mathrm{Fc} \gamma \mathrm{RIIB}$ that support their concept, they failed to discuss these in the context of the more recent contradictory findings. Thus, the mechanisms of action of IVIG across various murine models are not always identical and they depend on the genetic background and the dose of IVIG.

Additional studies in animal models of autoimmune diseases, such as lupus, antiphospholipid syndrome (APS), myasthenia gravis and pemphigus vulgaris, have revealed Fc-sialylation-independent and $\mathrm{F} c \gamma \mathrm{R}$-independent mechanisms of IVIG action that are not in line with the Fc-sialylation concept ${ }^{9,10}$. For instance, treating $\mathrm{NZB} \times \mathrm{W} / \mathrm{F} 1$ lupus-prone mice with a specific subfraction of IVIG (sIVIG) comprising anti-idiotypic antibodies specific for double-stranded DNA (dsDNA)-specific antibodies was 200 -fold more effective in reducing lupus glomerulonephritis than treating the mice with regular IVIG devoid of the anti-idiotypic antibodies, pointing to the importance of the $\mathrm{F}\left(\mathrm{ab}^{\prime}\right){ }_{2}$ portion of the sIVIG in this scenario. Moreover, treatment with sIVIG containing anti-idiotypic antibodies specific for $\beta 2$-glycoprotein 1 $(\beta 2 \mathrm{GP} 1)$-specific antibodies, but not treatment with the Fc portion of sIVIG, significantly improved the pregnancy outcome in $\mathrm{BALB} / \mathrm{c}$ mice that were passively infused with $\beta 2$ GP1-specific antibodies as compared with mice that received whole IVIG. Future studies will be required to test the validity of the Fc-sialylation concept in specific and distinct models of autoimmune disorders.

Further studies that propose alternative explanations of IVIG actions, as they include Fc-sialylation-independent and Fc $\gamma \mathrm{R}$ independent mechanisms, are inaccurately discussed by Schwab and Nimmerjahn ${ }^{1}$. Functional antibodies against FAS (also known as CD95 and TNFRSF6), sialic acidbinding Ig-like lectin 8 (SIGLEC8), and SIGLEC9 receptors that exhibit the capacity to regulate the survival of granulocytes and other leukocytes have been found in IVIG preparations ${ }^{11}$. Schwab and Nimmerjahn claim that IVIG infusion into patients and mice does not usually result in a significant reduction or depletion of these cell types. However, they fail to mention the fact that cytokine-primed granulocytes, such as those that are present at the site of inflammation, are highly susceptible to such deathpromoting antibodies, whereas resting cells are comparably resistant ${ }^{11}$. Furthermore, several reports by clinicians document a reduction in circulating neutrophils or eosinophils following infusion of IVIG in certain patients, which is probably as a 
result of exposure to priming factors, such as inflammatory mediators ${ }^{11}$. While Schwab and Nimmerjahn suspect that antibody titres in IVIG might be too low, it was shown that such antibodies occur at sufficient levels for isolation - by analogy to reports of antiidiotypic antibodies - and that they are of high affinity with the capacity to bind to leukocyte surfaces at detectable levels, as assessed by flow cytometry. Lower concentrations of functional antibodies in IVIG might explain the requirement of high-dose IVIG application for anti-inflammatory treatment. While the authors note that the "predicted" skin inflammation exacerbation is not observed through the presence of agonistic FAS-specific antibodies in IVIG, they conveniently overlook to 'predict' that the pro-inflammatory cells, including $\mathrm{T}_{\mathrm{H}} 1$ and $\mathrm{T}_{\mathrm{H}} 17$ cells, may undergo apoptosis through agonistic anti-FAS antibodies. Schwab and Nimmerjahn also discuss the occurrence of antagonistic FAS-specific antibodies in IVIG preparations, but they do not mention that the resulting effects between agonistic and antagonistic antibodies are concentration dependent; at low IVIG concentrations the protective effects of antagonistic antibodies prevail, which is consistent with the lack of deleterious effects of diluted IVIG on peripheral tissues ${ }^{11}$. Furthermore, although anti-idiotypic antibodies against SIGLEC9-specific or FAS-specific antibodies reside as anti-idiotypic complexes in dimeric IVIG, this does not mean, as postulated by Schwab and Nimmerjahn, that the activity of these antibodies is inhibited, because dimeric IgG rapidly dissociates following injection of IVIG due to temperature effects, concentration (dilution) and $\mathrm{pH}$, which results in the release of specific antibodies from anti-idiotypic complexes ${ }^{12}$.
Taken together, the mechanisms of action of IVIG involve a wide spectrum of Fabmediated and, probably, distinct Fc-mediated mechanisms, that may or may not depend on IVIG sialylation. The effects observed in various murine models may not be consistent and many of the disease-specific mechanisms must be validated in humans, as animal models only offer a limited insight into human disease and might be biased by xenogeneic or species-specific properties of IVIG ${ }^{11,13-15}$. IVIG is a pluripotent drug; its complexity, together with the pathogenetic heterogeneity of autoimmune diseases, remains a challenge to the scientist and does not allow for a simplistic perspective on its modes of action.

Stephan von Gunten and Hans-Uwe Simon are at the Institute of Pharmacology, University of Bern, CH-3010 Bern, Switzerland.

Miri Blank and Yehuda Shoenfeld are at the Zabludowicz Center for Autoimmune Diseases, Sheba Medical Center, Tel Aviv University, Tel Aviv 5262225, Israel. Yehuda Shoenfeld is also at the Incumbent of the Laura Schwarz-Kipp Chair for Research of Autoimmune Diseases, Tel Aviv University, Tel Aviv 5262225, Israel.

Donald R. Branch is at the Department of Medicine, University of Toronto, and Centre for Innovation, Canadian Blood Services, Toronto, Ontario M5G 2M1,

Canada.

Tchavdar Vassilev is at the Department of Immunology, Stefan Angelov Institute of Microbiology, Bulgarian Academy of Sciences, Sofia 1113, Bulgaria.

Fabian Käsermann is at Research and Development, CSL Behring AG, CH-3000 Bern, Switzerland.

Jagadeesh Bayry and Srini Kaveri are at Unité 1138, Institut National de la Santé et de la Recherche Médicale, Paris, France; Université Pierre et Marie Curie, Paris 6, Centre de Recherche des Cordeliers, 75006 Paris, France.

All authors contributed equally to this work. Correspondence to S.v.G. e-mail: stephan.vongunten@pki.unibe.ch doi: 10.1038/nri3401-c1
1. Schwab, I. \& Nimmerjahn, F. Intravenous immunoglobulin therapy: how does IgG modulate the immune system? Nature Rev. Immunol. 13, 176-189 (2013).

2. Abe, J. et al. Gene expression profiling of the effect of high-dose intravenous Ig in patients with Kawasaki disease. J. Immunol. 174, 5837-5845 (2005).

3. Maddur, M. S. et al. Inhibition of differentiation, amplification, and function of human $\mathrm{T}_{H} 17$ cells by intravenous immunoglobulin. J. Allergy Clin. Immunol. 127, 823-830 (2011).

4. Trinath, J. et al. Intravenous immunoglobulin expands regulatory $\mathrm{T}$ cells via induction of cyclooxygenase-2-dependent prostaglandin E2 in human dendritic cells. Blood 122, 1419-1427 (2013).

5. Käsermann, F. et al. Analysis and functional consequences of increased Fab-sialylation of intravenous immunoglobulin (IVIG) after lectin fractionation. PLOS ONE 7, e37243 (2012).

6. Guhr, T. et al. Enrichment of sialylated IgG by lectin fractionation does not enhance the efficacy of immunoglobulin $\mathrm{G}$ in a murine model of immune thrombocytopenia. PLOS ONE 6, e21246 (2011).

7. Leontyev, D. et al. Sialylation-independent mechanism involved in the amelioration of murine immune thrombocytopenia using intravenous gammaglobulin. Transfusion 52, 1799-1805 (2012).

8. Bazin, R., Lemieux, R. \& Tremblay, T. Reversal of immune thrombocytopenia in mice by cross-linking human immunoglobulin $\mathrm{G}$ with a high-affinity monoclonal antibody. Br. J. Haematol. 135, 97-100 (2006).

9. Leontyev, D., Katsman, Y. \& Branch, D. R. Mouse background and IVIG dosage are critical in establishing the role of inhibitory Fc $\gamma$ receptor for the amelioration of experimental ITP. Blood 119, 5261-5264 (2012).

10. Svetlicky, N. et al. The advantage of specific intravenous immunoglobulin (SIVIG) on regular IVIG experience of the last decade. J. Clin. Immunol. 33, S27-S32 (2013).

11. von Gunten, S. \& Simon, H. U. Cell death modulation by intravenous immunoglobulin. J. Clin. Immunol. 30 (Suppl. 1), 24-30 (2010).

12. Durandy, A. et al. Intravenous immunoglobulins understanding properties and mechanisms. Clin. Exp. Immunol. 158 (Suppl. 1), 2-13 (2009).

13. Gelfand, E. W. Intravenous immune globulin in autoimmune and inflammatory diseases. N. Engl. J. Med. 367, 2015-2025 (2012).

14. Seok, J. et al. Genomic responses in mouse models poorly mimic human inflammatory diseases. Proc. Natl Acad. Sci. USA 110, 3507-3512 (2013).

15. Trinath, J., Hegde, P., Balaji, K. N., Kaveri, S. V. \& Bayry, J. Intravenous immunoglobulin-mediated regulation of Notch ligands on human dendritic cells. J. Allergy Clin. Immunol. 131, 1255-1257 (2013).

Competing interests statement

The authors declare no competing interests. 